\title{
Two methods to measure granular gas temperature.
}

\author{
J.-Y. Chastaing, J.-C. Géminard, A. Naert \\ Univ Lyon, Ens de Lyon, Univ Claude Bernard, CNRS, Laboratoire de Physique, \\ F-69342 Lyon, France \\ E-mail: Antoine.Naert@ens-lyon.fr
}

\begin{abstract}
Grains are vibrated so as to achieve a granular gas, here regarded as an archetype for a dissipative Non Equilibrium Steady State (NESS). We report on two distinct and concordant experimental measures of the system effective temperature. To do so, a blade fastened to the shaft of a small DC-motor, immersed in the grains, behaves as a driven 1D Brownian rotator which is used as both actuator and sensor, simultaneously. On the one hand, the Gallavotti-Cohen Fluctuation Theorem (FT), which involves a measure of the asymmetry of the energy exchanges between the rotator and the NESS reservoir, provides a first effective temperature. On the other hand, the Fluctuation-Dissipation Theorem (FDT), which involves the relation between the spontaneous fluctuations and the response to a weak perturbation, defines a second, independent, effective temperature. Both methods, even though they are based on drastically different ideas, give nicely concordant results.
\end{abstract}

\section{Introduction}

The interpretation of the velocity fluctuations of a granular flow in terms of 'temperature' appeared relatively late in the study of granular matter, in the end of the seventies (see for instance [1], and other references in the review papers by Goldhirsch $[2,3])$. The reason is probably that former studies of granular matter concentrated on dense phases, mainly for the purpose of soil mechanics, in which velocity fluctuations are not relevant. In the limit case of a granular packing at rest, a temperature, based on the concept of the entropy of configuration, has been introduced $[4,5,6]$. In the present article, we deal with dynamical situations in which velocity fluctuations play a fundamental role. The theoretical idea of introducing a temperature, inspired from statistical mechanics, was very soon put to use in constructing kinetic theories of granular gases, since the beginning of the eighties. The study of granular matter from the viewpoint of kinetic and hydrodynamic theories has been improved since then, in most of the cases by relying on the concept of granular temperature [7]. One must note that the granular temperature, defined as the (ensemble) average of the squared velocity fluctuations, differs from the internal (thermodynamic) temperature of the grains. Nevertheless, it characterizes the fluctuations of the grains velocity, alike the thermodynamic temperature in molecular liquids or gases. 
Two methods to measure granular gas temperature.

Although theories developed since then use extensively the concept of granular temperature, this quantity remains difficult to assess. Video tracking can be used, but only in 2D flows [8]. In 3D however, high-end techniques have been used such as positron-emission particle tracking [9] or magnetic resonance [10]. But, in general, the comparison of theories is possible only with numerical simulations.

We propose in this article to investigate the temperature indirectly. As indirect temperature measurement, one can consider a forced Brownian-like harmonic oscillator in a fluidized granular bath, which gives access to granular viscosity and effective temperature [11], thanks to the Fluctuation-Dissipation Theorem (FDT) [12]. Another experiment based on an harmonic oscillator in a rarefied granular gas makes use of the Gallavotti-Cohen Fluctuation Theorem [13, 14] to define and measure a granular

effective temperature [15]. Actually, both studies suppose that the oscillating object is thermalized with the bath. A recent study showed that this hypothesis deserves longer discussion, especially for very low densities [16]. A different, indirect, approach was introduced in simulations of a 2D granular gas by Aumaître et al. [17]. These authors investigate the fluctuations of the power provided to the granular gas to compensate dissipation. Thanks to the FT again, they define and measure a global effective granular temperature, proportional to the mean kinetic energy of the beads. Just to mention, the specific case of a single grain can be analyzed the same way [18, 19].

The present article reports on simultaneous measurements of two effective temperatures in a rarefied granular gas, thanks to the heuristic use of the FDT on the one hand, and of the FT on the other hand. To perform measurements, we make use of a small DC motor, that can be used as both sensor and actuator. For the FDT-based measurements, we present an original spectral method, that makes possible to obtain simultaneously the free fluctuations and the susceptibility to an imposed perturbation. The method provides a first estimate of the granular temperature. For comparison, a second estimate of the granular temperature is obtained by using the same set of data and a FT-based method.

\section{Methods}

We propose in Fig. 1a a sketch of the experimental setup. The granular gas is composed of $N \simeq 340$ stainless steel beads of diameter $3 \mathrm{~mm}$ and mass $m \simeq 0.1 \mathrm{~g}$ placed in an aluminum vessel $(5 \mathrm{~cm}$ in diameter and $6 \mathrm{~cm}$ in height). The vessel, whose inner bottom is slightly cone-shaped (angle $\simeq 10^{\circ}$ ) in order to favor the transfer from vertical to horizontal momentum, is vibrated vertically. The electromechanical shaker (Brüel \& Kjær, 4809), which insures the vibration, is fed with the current from a power amplifier (Kepco, BOP 36-6M) driven by a sine at $40 \mathrm{~Hz}$ from a waveform generator (Keysight, 35500B). Changing the excitation power (of a few Watts), we tune the maximum vertical acceleration of the vessel which is always of a few times the acceleration due to gravity. In the stationary regime, the granular gas is in a dissipative Non-Equilibrium Steady State (NESS) as the energy provided by the 
shaker compensates the continuous energy losses (mainly due to the collisions). In the following, this steady-state granular-gas will be regarded as the thermostat over which measurements are performed.
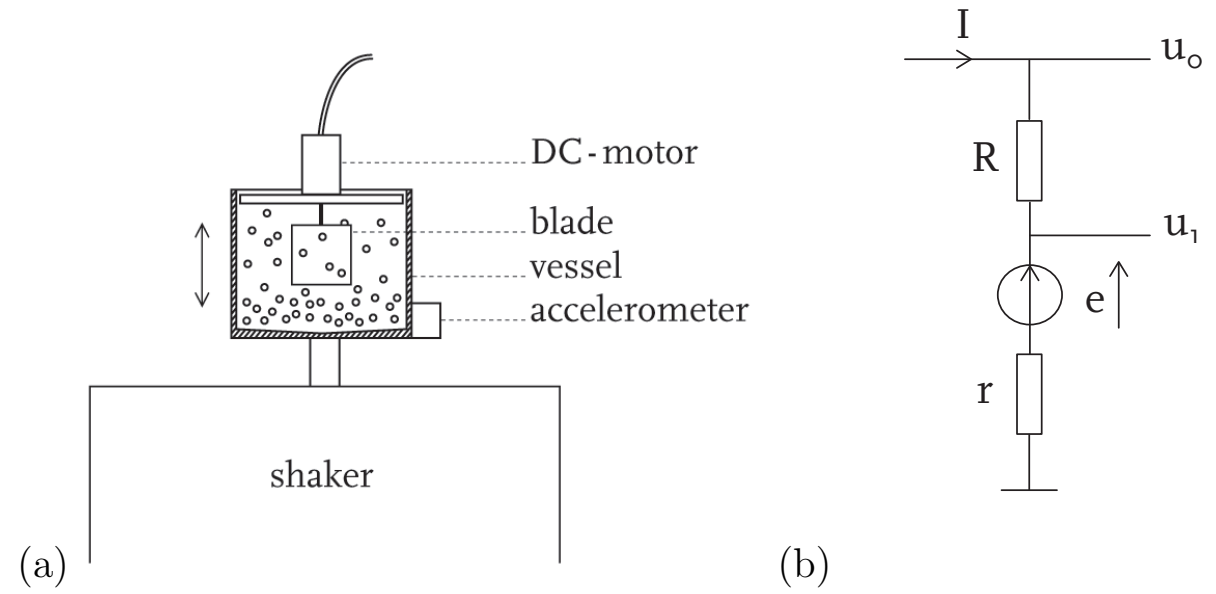

(b)

Figure 1. (a) Sketch of the experimental setup - The grains, placed in a vessel, are vibrated vertically by means of an electomechanical shaker. A blade fastened to the shaft of a small DC-motor, immersed in the grains, behaves as a driven 1D Brownian rotator which is used as both actuator and sensor, simultaneously. (b) Electrical circuit associated to the probe - The motor is modeled by the combination of an ideal voltage source $e(\propto \dot{\theta}$, the angular velocity of the blade) and of an internal resistance $r$.

A steel blade $\left(20 \times 20 \mathrm{~mm}^{2}\right.$, thickness $\left.0.5 \mathrm{~mm}\right)$, fastened to the shaft of a DC micro-motor (Maxon RE 10 118386), immersed in the gas, will be our sensor (Fig. 1a). The micro-motor (10 $\mathrm{mm}$ in diameter) is attached to the upper cover of the vessel, its shaft being vertical, along the symmetry axis of the setup. Concerning the mechanical characteristics of the sensor, the moment of inertia of the $\{$ rotor + blade $\}$ ensemble is $J \simeq 3.3310^{-8} \mathrm{~kg} \mathrm{~m}^{2}$. Concerning its electrical characteristics, the micro-motor is a permanent magnet, coreless, brushed DC motor of nominal power $0.75 \mathrm{~W}$ and nominal voltage $6 \mathrm{~V}$, exhibiting an internal resistance of $r \simeq 21.2 \Omega$.

The original and fruitful feature of our experiment relies on the astute way to make use of the electromechanical symmetry of the DC motor. Indeed, a DC motor is usable as both motor and generator, reversibly. When it is used as a motor, a current $I$ is converted into a mechanical torque $\Gamma=\alpha I$ applied to the rotor. When it is used as a generator, an angular velocity $\dot{\theta}$ of the rotor results in a voltage difference $e=\alpha \dot{\theta}$ between the terminals. The key point for our study is that the same coefficient $\alpha$ couples the torque and the current as a motor, and the voltage and the angular velocity as a generator. Because of this peculiar property, in the following, the same device is used as both actuator and sensor without any need of calibration.

During the experiments, the blade is immersed in the granular gas and the motor is fed, through a resistor $R=23 \Omega$, with the current $I(t)$ from a programmable wave 
generator (Agilent 33522A, Fig. 1b). At the same time, the voltages $u_{0}$ and $u_{1}$ at the terminals of $R$ are recorded simultaneously by a 24-bit acquisition board (NI-PXI 4462) at sampling frequency $f_{s}=1024 \mathrm{~Hz}$. On the one hand, the current $I$ supplied to the motor induces a torque $\Gamma$ that tends to rotate the blade in the granular gas. At any time $t, I(t)$ is obtained from $u_{0}(t)$ and $u_{1}(t)$ by using the relation $I=\left(u_{0}-u_{1}\right) / R$. An angular velocity $\dot{\theta}(=e / \alpha)$ results from the combined effects of the torque $\Gamma$ and of the interaction with the gas. We get $e(t)$ from the measured voltages $u_{0}(t)$ and $u_{1}(t)$ by using the relation $e=u_{1}-r I$ (The internal resistance of the motor, $r$, must be precisely known. We checked that the effects of the inductance of the motor, $L \simeq 0.184 \mathrm{mH}$, are negligible in our experimental conditions).

(a)

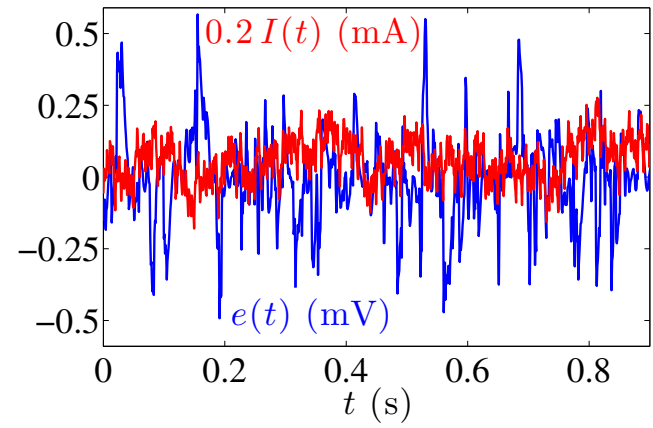

(b)

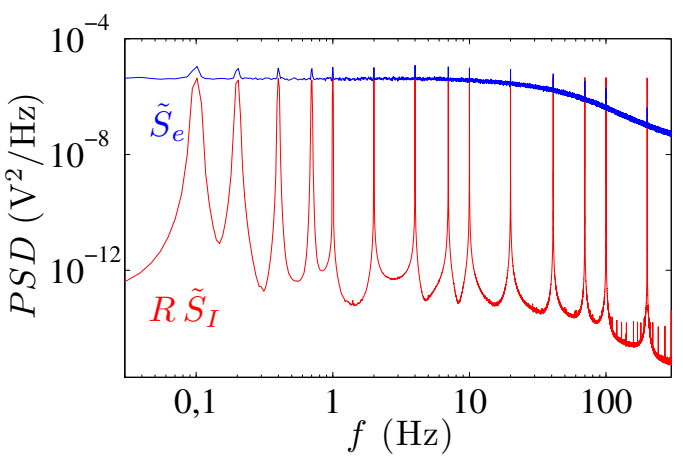

Figure 2. (a) Time series of: Red, the injected current $I(t)(\mathrm{mA})$; blue, the voltage $e(t)(\mathrm{V})$. (b) Corresponding power spectral density (PSD) of: Red, $I(t)$. The 15 chosen frequencies are clearly revealed by the large peaks; Blue: $e(t)$. The injected current $I(t)$ results in the appearance of 15 tiny peaks above the base line which corresponds to the free fluctuations (Acceleration $a=2.7 \mathrm{~g}$ ).

The idea is to assess simultaneously the free fluctuations of the blade and the response to a perturbation. In absence of imposed current $I(t)$, the voltage $e(t)$ fluctuates because of the collisions between the grains and the blade. In order to assess the response to a perturbation, we impose that the current $I(t)$ follows a periodic pattern, consisting of the sum of 15 sine functions of different frequencies $f_{e}$, logarithmically distributed over the range $0.1 \leq f_{e} \leq 400 \mathrm{~Hz}$ (Fig. 2). This range covers the low-frequency flat-part of the Lorentzian-like spectrum of the spontaneous fluctuations of the blade velocity, and goes beyond the corner frequency $\omega_{c}$ above which the amplitude of the spontaneous fluctuations significantly decreases (Fig. 2b). In addition, in order to ensure that we only weakly perturb the system, we choose the amplitude of each of the sine functions such that its contribution appears as peak of small relative amplitude in the power spectrum (Fig. 2b). Finally, the relative phases of the different components are randomized, such as to avoid stiff variation of the current caused by unfortunate interferences.

After the experiment is started, a few hours are necessary to reach a stationary regime (constant variances). The internal resistance $r$ of the motor is measured both before and after the experiment in order to detect undesired drifts and to ensure the 
Two methods to measure granular gas temperature.

use of the appropriate value when calculating $e$. The experimental results are obtained from the adequate analysis of the temporal series of the voltages $u_{0}$ and $u_{1}$, recorded during 20 to $40 \mathrm{~h}$ in order to collect enough statistics.

\section{Experimental results}

\subsection{Energy exchanges: effective temperature from the Fluctuation Theorem}

In this first experimental section, we use the Fluctuation Theorem (FT) to estimate a first effective temperature, as we previously did for a similar granular system [15].

Consider the mechanical power exchanged between the blade and the granular gas, $\dot{w} \equiv \Gamma \dot{\theta}$. Noticing that $\dot{w}=I e$ in terms of current $I$ and voltage $e$ (Sec. 2), we easily get the temporal series of $\dot{w}$ from the set of data $\left\{u_{0}, u_{1}\right\}$ obtained experimentally. The power $\dot{w}$, although positive on the average because of the dissipative nature of the system, presents frequent negative fluctuations. We define the coarse-grained work rate $\dot{w}_{\tau}(t)=\frac{1}{\tau} \int_{t}^{t+\tau} e\left(t^{\prime}\right) I\left(t^{\prime}\right) d t^{\prime}$ (the average over the time $\tau$ ) and denote $P$ the probability density function of this new variable. In our experimental conditions, the probability of negative $\dot{w}_{\tau}$ remains non zero for all $\tau$.

(a)

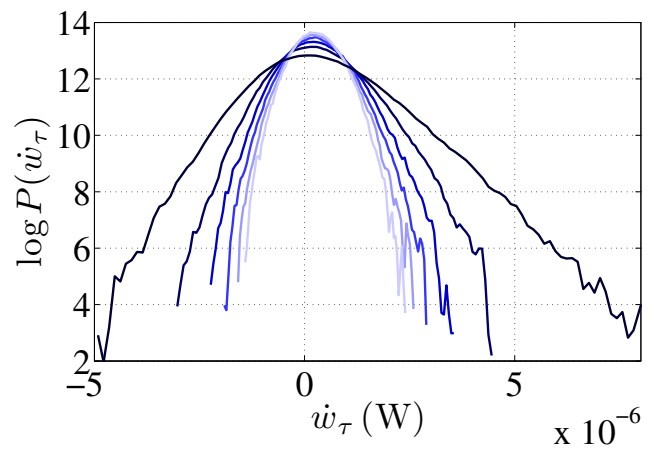

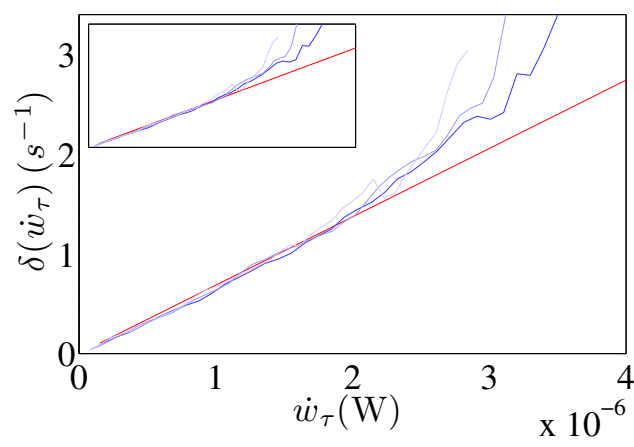

Figure 3. (a) Probability density function $P$ of the power $\dot{w}_{\tau}$ - The time $\tau$ is increased from $10 \mathrm{~s}$ to $60 \mathrm{~s}$ by steps of $10 \mathrm{~s}$, from black to light gray. The mean power exchanged is here $\left\langle\dot{w}_{\tau}\right\rangle=2.510^{-7} \mathrm{~W}$. (b) Corresponding asymmetry function $\delta$. The continuous line shows the asymptotic slope at the origin (Acceleration $a=2.7 \mathrm{~g}$ ).

For a more quantitive characterization of the asymmetry of the energy exchanges, let us consider the asymmetry function $\delta\left(\dot{w}_{\tau}\right) \equiv \frac{1}{\tau} \log \frac{P\left(\dot{w}_{\tau}\right)}{P\left(-\dot{w}_{\tau}\right)}$. The Fluctuation Theorem (FT), which accounts for the irreversibility of the exchanges for a chaotic system in contact with an equibrium thermostat at temperature $T$ through a relation for the rate of entropy production $\dot{s}_{\tau} \equiv \dot{w}_{\tau} / T$, states that, in the limit of large $\tau$ :

$$
\frac{P\left(\dot{w}_{\tau}\right)}{P\left(-\dot{w}_{\tau}\right)}=e^{\beta \tau \dot{w}_{\tau}}
$$

In this framework, the well-defined exponent $\beta=1 / k_{\mathrm{B}} T$, where $k_{\mathrm{B}}$ is the Boltzmann constant $\left(k_{\mathrm{B}} \simeq 1.3810^{-23} \mathrm{~J} \mathrm{~K}^{-1}\right)$ and $T$ is the temperature of the reservoir $[13,14]$. In Fig. 3b, we display $\delta\left(\dot{w}_{\tau}\right)$ for various $\tau$. We observe that $\delta\left(\dot{w}_{\tau}\right)$ is almost proportional 
to $\dot{w}_{\tau}$ such that Eq. (1) is almost satisfied by our system. Letting aside the question of the applicability of the FT when the reservoir is a NESS, we adjust the asymmetry function $\delta\left(\dot{w}_{\tau}\right)$ to a line over the lower $3 / 4$ of the whole range of $\dot{w}_{\tau}$ (statistically the most relevant). We report in Fig. 4, the inverse of the slope $1 / \beta$ against the time $\tau$ and observe that $1 / \beta$ reaches a plateau at large $\tau$. We interpret the plateau value as the effective temperature $k T_{\text {eff. }}$ of the granular gas, which plays the role of the thermostat in our experimental configuration. Note that, the Boltzmann constant being irrelevant for our macroscopic system, we consider here an energy scale $k T_{\text {eff. }}$ rather than a temperature. This estimate of the effective temperature of the thermostat being obtained by the use of the FT will be later denoted $k T_{\mathrm{FT}}$.

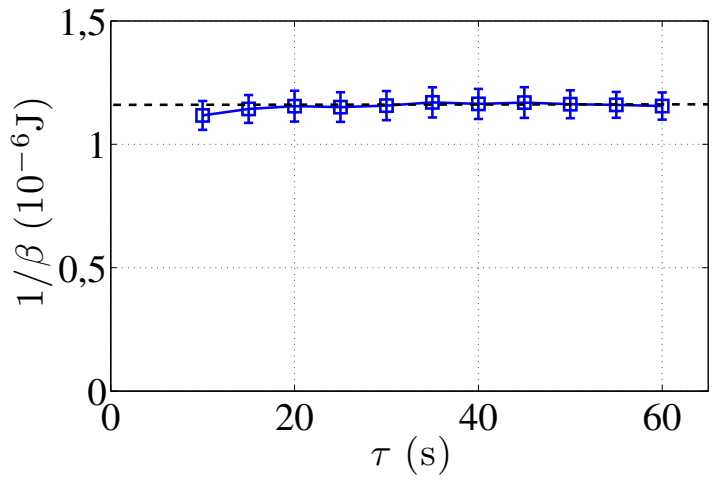

Figure 4. Inverse of the slope $1 / \beta$ vs. time $\tau$. We consider the value of $1 / \beta$ reached at large $\tau$ (dashed line) as an estimate of the effective temperature $k T_{\text {eff. }} \simeq 1.1510^{-6} \mathrm{~J}$ of the granular gas which plays the role of the thermostat in our experimental configuration (Acceleration $a=2.7 \mathrm{~g}$ ).

\subsection{Response to a perturbation: the Fluctuation-dissipation relation}

The Fluctuation-Dissipation Theorem (FDT) has a seminal importance in statistical mechanics, specifically in the framework of linear response theory. It is based on the assumption that the response of a system, slightly driven off-equilibrium, relaxes in the same way whether the cause is a spontaneous internal fluctuation or an external perturbation. The FDT states that the two-point correlation function $C(\tau)=\langle\theta(t+$ $\tau) \theta(t)\rangle_{t}$ of any extensive variable $\theta$ is proportional to the response function $\mathcal{R}(\tau)$ of the variable $\theta$ to its conjugate intensive variable $\Gamma$. To a numerical constant, the prefactor is nothing but the equilibrium temperature of the thermostat. The FDT thus provides an absolute definition of the temperature under a twofold equilibrium hypothesis: the system under consideration is in equilibrium with the thermostat, and the thermostat itself is in equilibrium [12]. Note that, if the two conjugate variables are both independently accessible to experiment, verifying the FDT gives an operational way to measure temperature. Cugliandolo and Kurchan proposed in 1997 to extend this approach to off-equilibrium systems, like slowly relaxing (quasi steady state) or driven 
(steady state) systems, in order to define a non-equilibrium effective temperature [20]. This method has been implemented experimentally in a variety of non-equilibrium situations such as amorphous materials below the glass transition [21, 22], jammed granular materials [23], systems close to a critical point [24], soft matter [25], turbulence $[26,27]$ and, even, active matter [28].

Let us consider our experimental configuration where the system is an externally driven rotator immersed in a granular gas. The two conjugate variables relevant for this system are the torque $\Gamma$ and the angle $\theta$. We write the angle $\theta(t)$ as the response to the torque $\Gamma: \theta(t)=\int_{-\infty}^{t} \mathcal{R}\left(t-t^{\prime}\right) \Gamma\left(t^{\prime}\right) d t^{\prime}$. In the Fourier space, $\tilde{\mathcal{R}}=\tilde{\theta} / \tilde{\Gamma}$ where . denotes Fourier transform. Introducing the power spectral density (PSD) $\tilde{S}_{\theta}^{2} \equiv\left\langle|\tilde{\theta}|^{2}\right\rangle$, which equals the Fourier transform of the correlation function $C(\tau)=\langle\theta(t+\tau) \theta(t)\rangle_{t}$, we can express the fluctuation dissipation relation in the form:

$$
\frac{\tilde{S}_{\theta}^{2}}{4 \operatorname{Im}(\tilde{\mathcal{R}})}=k T_{\text {eff. }}
$$

The imaginary part of the response, $\operatorname{Im}(\tilde{\mathcal{R}})$, that appears in the denominator stands for the dissipative part of the response. Eq. (2) would express the FDT, if it were applying. However, in the present case, the product $k_{\mathrm{B}} T$ of the Boltzmann constant $k_{\mathrm{B}}$, and thermostat temperature $T$, which is to be considered for an equilibrium system, is replaced by the effective temperature, $k T_{\text {eff. }}$. We remark that, in our experimental configuration, the observables are the torque $\Gamma$ and the angular velocity $\dot{\theta}$ of the rotor, not the angular position $\theta$. The fluctuation-dissipation relation can be reformulated in these variables by taking into account the response $\tilde{\mathcal{R}}^{\prime}=\tilde{\dot{\theta}} / \tilde{\Gamma}$, instead of $\tilde{\mathcal{R}}$. To do so, the imaginary part $\operatorname{Im}(\tilde{\mathcal{R}})$ of the response $\tilde{\mathcal{R}}$, which accounts for the dissipation in Eq. (2), is now represented by the real part $R e\left(\tilde{\mathcal{R}}^{\prime}\right)$ of the response $\tilde{\mathcal{R}}^{\prime}$. In addition, considering that the torque $\Gamma$ and the angular velocity $\dot{\theta}$ are proportional to the current $I$ and voltage $e$, respectively, we write:

$$
k T_{\text {eff. }}=\frac{\tilde{S}_{e}^{2}}{4 \operatorname{Re}(\tilde{e} / \tilde{I})} .
$$

Note that, thanks to the reversibility of the probe, the calibration factor $\alpha$ cancels out. In other words, the measure of the granular temperature $k T_{\text {eff. }}$ does not require any calibration of the probe. In what follows, we describe how the response and the fluctuations are assessed in practice, separately but from the same data set, in order to

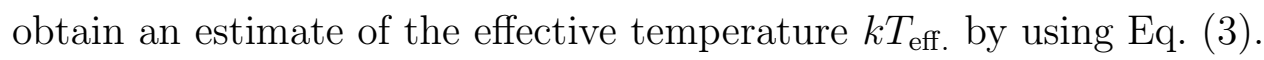

3.2.1. Response function - The response function is obtained thanks to the imposed current $I(t)$ (Sec. 2). We remind here that the current $I(t)$ follows a periodic pattern, consisting of the sum of 15 sine functions of different frequencies $f_{e}$, logarithmically distributed over the range $0.1 \leq f_{e} \leq 400 \mathrm{~Hz}$ (Fig. 2). The voltage $e(t)$ exhibits a response at each of the frequencies $f_{e}$, such that we get estimates of the response function, $\tilde{e} / \tilde{I}$ or equivalently of $\tilde{\mathcal{R}}^{\prime}$, in 15 points (Fig. 5). 
(a)

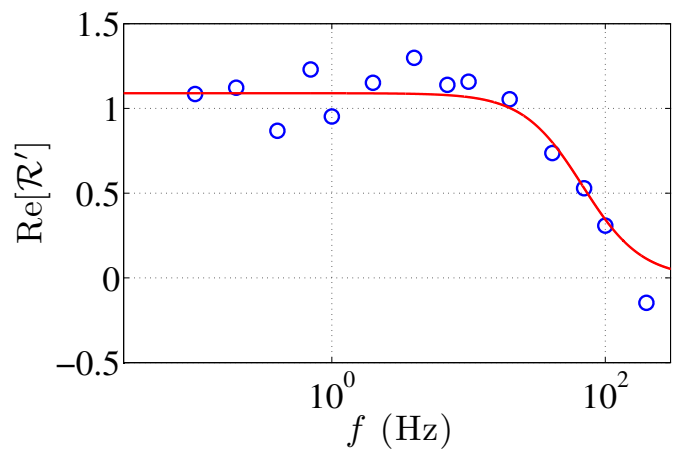

(b)

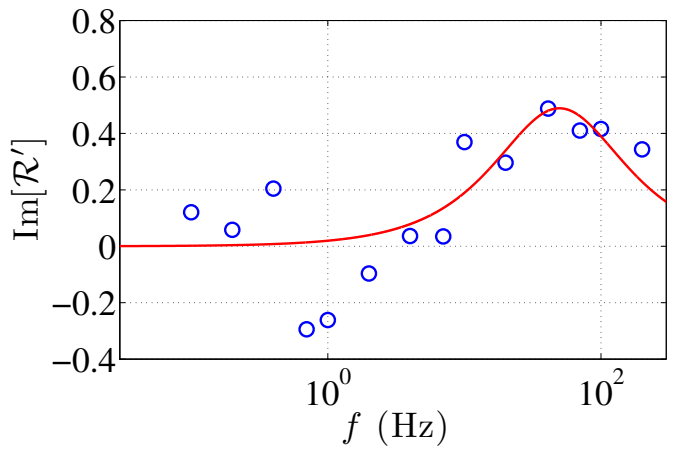

Figure 5. (a) Real $\operatorname{Re}\left(\tilde{\mathcal{R}}^{\prime}\right)$ and (b) imaginary $\operatorname{Im}\left(\tilde{\mathcal{R}}^{\prime}\right)$ parts of the response function $\tilde{\mathcal{R}}^{\prime}$ vs. frequency $f$-Circles: values directly measured at the frequencies $f_{e}$ [Lines: interpolation to Eq. (5). Acceleration $a=2.7 \mathrm{~g}]$.

The response is noisy and, in order to get a better estimate, we model our mechanical system. We first assume that the dynamics of the rotator obeys:

$$
J \frac{d \dot{\theta}}{d t}=-\gamma \dot{\theta}+\Gamma(t)+\eta(t)
$$

where $J$ is the moment of inertia of the $\{$ blade + rotor $\}$ ensemble. The blade experiences a drag, assumed to be viscous, $-\gamma \dot{\theta}$, due to the combined effects of the interaction with the granular gas and of the internal friction in the motor. The blade undergoes the random forcing due to the collisions with the grains, $\eta(t)$. In addition, the blade is subjected to the torque $\Gamma(t)$, associated with the imposed current $I(t)$. This formulation is directly inspired by the theory of Brownian motion, where the interaction between a Brownian colloid and the surrounding molecules is represented by the sum of a viscous drag and of a random forcing, in the same equation of motion.

In the Fourier space, from Eq. (4), we easily get the response function $\tilde{\mathcal{R}}^{\prime}$, whose real and imaginary parts can be written in the form:

$$
\operatorname{Re}\left(\tilde{\mathcal{R}}^{\prime}\right)=\frac{1 / \gamma}{1+\left(\omega / \omega_{c}\right)^{2}} \quad \text { and } \quad \operatorname{Im}\left(\tilde{\mathcal{R}}^{\prime}\right)=\frac{\omega / \gamma \omega_{c}}{1+\left(\omega / \omega_{c}\right)^{2}},
$$

where $\omega=2 \pi f$ is the angular frequency and $\omega_{c}=\gamma / J$ the inverse of the damping time. The interpolation of the experimental data with Eq. (5), the sole adjustable parameter being $\gamma\left(J\right.$ is known), leads to a reasonnable agreement, specifically for $\operatorname{Re}\left(\tilde{\mathcal{R}}^{\prime}\right)$ which is the quantity of interest that appears in Eq. (3) (Fig. 5). In what follows, we will use the values of $\operatorname{Re}\left(\tilde{\mathcal{R}}^{\prime}\right)$ given by this interpolation of the experimental data.

3.2.2. Power spectral density - In order to determine the effective temperature by using Eq. (3), we need an estimate of the the power spectral density (PSD), associated to the free fluctuations of the blade, in particular at the frequencies $f_{e}$. However, at these specific frequencies, the system is forced. Considering that the PSD is not altered apart from the peaks, we interpolate the baseline of the power spectrum by a spline function and estimate the values of the PSD at the $f_{e}$ by continuity (Fig. 6). 


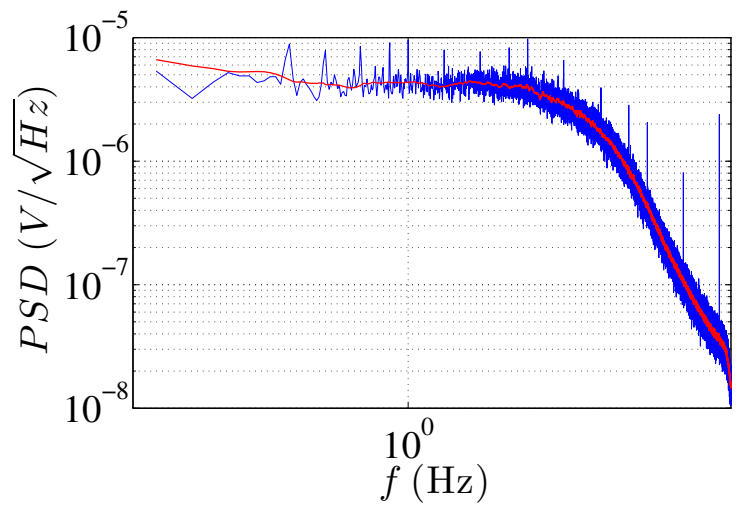

Figure 6. Power spectral density PSD of the voltage $e$ - The peaks correspond to the response to the imposed current $I$ at the frequencies $f_{e}$ (Blue: experimental data. Red: interpolation to a spline function apart from the frequencies $f_{e}$. Acceleration $a=2.7 \mathrm{~g})$.

3.2.3. Effective temperature - In the two previous sections, we have shown how to characterize, on the one hand the response function $\tilde{\mathcal{R}}^{\prime}$ and, on the other hand the power spectral density. We can thus now use Eq. (3), i.e. the fluctuation dissipation relation, to attempt to determine the effective temperature of the granular thermostat.

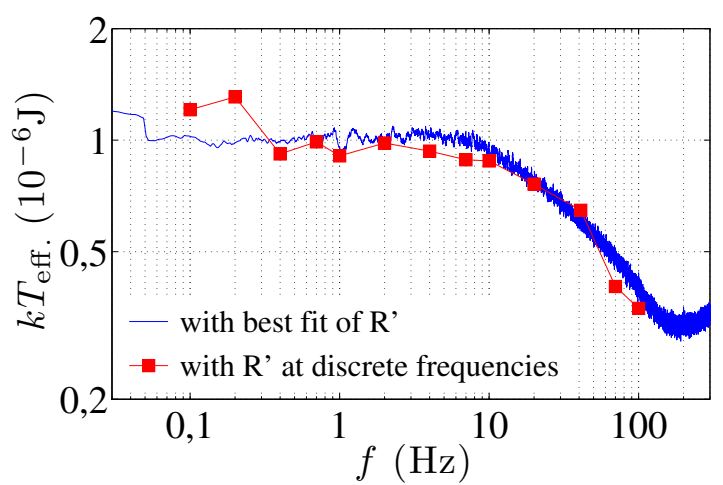

Figure 7. Ratio $\tilde{S}_{e}^{2} /[4 \operatorname{Re}(\tilde{e} / \tilde{I})]$ vs. frequency $f$ - The plateau value provides an estimate of the effective temperature of the thermostat $k T_{\text {eff. }} \simeq 10^{-6} \mathrm{~J}$. [Squares: experimental data obtained by using the values of the response function reported in Fig. 5. Points: data obtained by using the interpolation to Eq. (5). Acceleration $a=2.7 \mathrm{~g}$.

In Fig. 7, we report the ratio $\tilde{S}_{e}^{2} /[4 \operatorname{Re}(\tilde{e} / \tilde{I})]$, thus supposedly $k T_{\text {eff. }}$, versus the frequency $f$. We expect the quantity not to depend on $f$. Indeed, one can observe a plateau at small $f$, although a drastic decrease at large $f$ is observed. The decrease at large $f$ is due to the fact that the blade, because of its inertia, cannot fluctuate rapidly (Eq. 5). Nevertheless, the plateau value can be regarded as an estimate of the effective 
temperature of the thermostat $k T_{\text {eff. }}$. The latter, obtained by the use of the fluctuation dissipation relation, will be denoted $k T_{\mathrm{FDT}}$.

\section{Discussion}

We obtained two independent effective temperatures of the granular gas which we regarded as the thermostat in our study. First, we recalled and used a method based on the fluctuation theorem (Sec. 3.1). Second, we implemented and used a method based on the fluctuation dissipation theorem with the same set of data (Sec. 3.2). We remind that these two methods are based on drastically different principles. It is thus of particular interest to verify that the effective temperatures estimated from these two methods actually coincide. In Fig. 8, we observe that values of the effective temperature measured by one or the other method are equal to within $10 \%$ on the average. We remark however that estimated values of $k T_{\mathrm{FT}}$ are systematically larger than those $k T_{F D T}$. Even though we do not have any explanation for this trend, considering that the difference remains in the error bars and that the two methods are completely distinct, we conclude that the two estimates of the temperature nicely coincide.

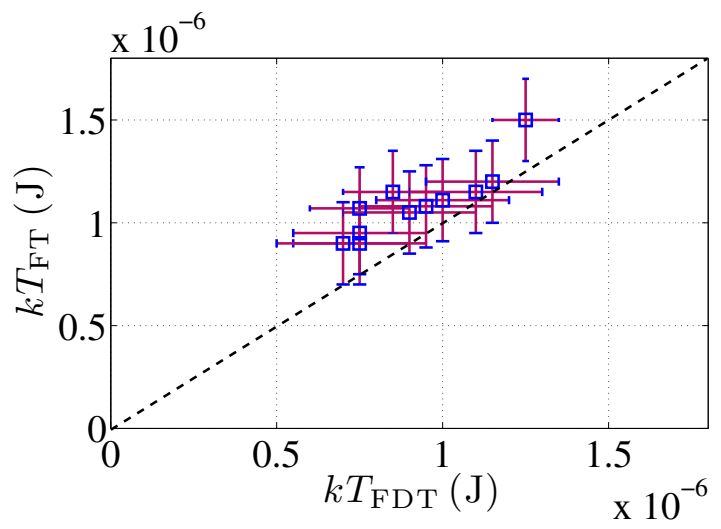

Figure 8. $k T_{\mathrm{FT}}$ vs. $k T_{F D T}$ - To within the experimental precision, the two estimates of the effective temperature coincide in the whole experimental range.

Still inspired by the phenomenology of Brownian Motion, one expects the

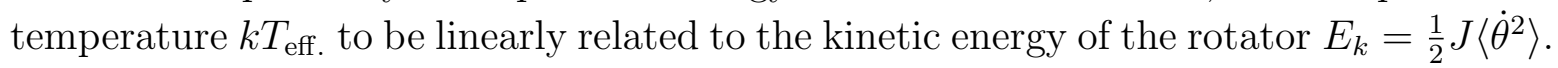
A linear dependance has already been reported in [15]. This expectation is checked in Fig. 9. We notice that the dashed line picturing the best linear fit, does not go through zero and has a slope of about 2.5. The error bars, a few percents, are evaluated as the remaining error in the fitting process for the method based on FT, and dominated by the statistical uncertainties for the other method. On the one hand, we conjecture that the value of $k T_{\text {eff. }}^{0}$ corresponding to $E_{k}=0$ is the temperature at which beads are barely energetic enough to reach the blade. Besides, no evident interpretation can be produced about the slope in such dissipative system. For a 1D-Brownian rotator in an 
equilibrium thermostat, one would expect a slope 2 from the equipartition of energy $\frac{1}{2} k T=\frac{1}{2} J\left\langle\dot{\theta}^{2}\right\rangle$. Note that we assess the fluctuations of the blade angular-position and, thus strictly speaking, the temperature of the probe which does not necessarily equals that of the granular gas (We specifically address this delicate issue, which goes beyond the present study, in [16]). The discrepancy, attributed to dissipation, however preserves the order of magnitude.

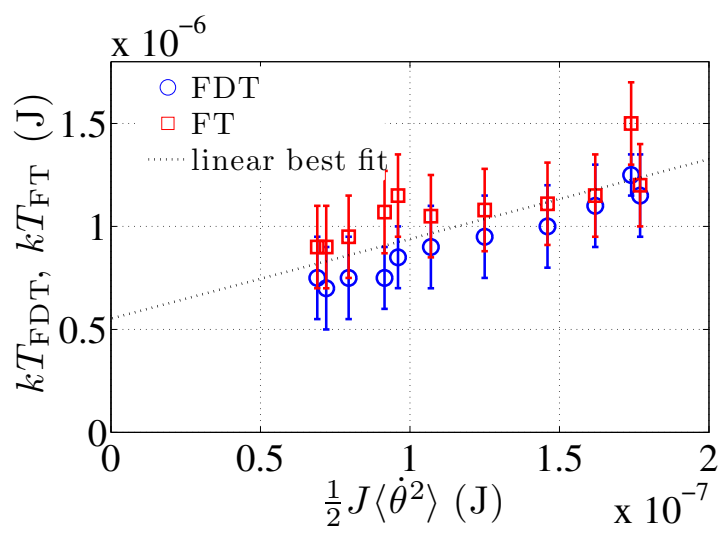

Figure 9. $k T_{\mathrm{FDT}}$ and $k T_{F T}$ vs. kinetic energy of the rotator.

At last, we must point out that both methods are not easy, experimentally. First, they require enough statistics to approach the long time-limit. Second, the probing power $\left\langle\dot{w}_{\tau}\right\rangle$ must remain small enough, on the one hand to favor negative events (FT), on the other hand not to perturb significantly the granular gas (FT and FDT). But, the experiments are worth as they prove that both methods lead to the same effective temperature.

\section{Conclusion}

We presented in this article an experiment using a few hundreds of beads, maintained in a gaseous steady state thanks to the external power supplied by a shaker. This granular gas possesses a large number of degrees of freedom, which guaranties an efficient chaotic loss of memory. It therefore mimics rather well a real gas, however dissipative (a NESS). As a probe, a small rotator is immersed in the granular gas, secured on the shaft of a vertically set micro-motor. Our experimental approach makes possible to measure the response of the system to a perturbation (at all frequencies), and, what is shrewd, the free fluctuations of the unperturbed system at the same time.

We made use of the experimental realization to estimate the effective temperature of the system based on the Fluctuation-Dissipation Theorem (FDT) and to compare the latter with another estimate obtained from an independent method, based on the GallavottiCohen Fluctuation Theorem (FT), using the same set of data. Both methods lead to the same value of the effective temperature (to within the experimental accuracy). From the theoretical viewpoint, the FDT can be derived from the FT, when the linear response is 
assumed. However, even if linearity is not assured in our experimental system, we can consider, from now on, that the measurements of the granular effective temperature are validated and, therefore, that the probe indeed constitutes a thermometer.

We note that in our macroscopic system, the fluctuations are very large compared to the mean energy exchanged between the probe and the reservoir. This is due to the fact that the number of degrees of freedom, although large, is far from the thermodynamic limit. This feature, which would appear in micro- or nano-systems, makes convenient these techniques based on measurements of fluctuations. Of course, as our system is dissipative, the analogy is to be considered with caution. But defining a consistent out-of-equilibrium temperature and showing operational procedures to measure it is definitely a step forward.

\section{Acknowledgments}

A.N. especially acknowledges S. Ciliberto, S. Aumaître, P. Cobelli and K. Mallick for discussions, and P. Petitjeans for welcome in PMMH.

[1] S. Ogawa. Multitemperature theory of granular materials. In S.C. Cowin, M. Satake (Eds.), Proc. U. S.-Japan Symp. on Continuum Mechanics and Statistical Approaches in the Mechanics of Granular Materials, Gakujutsu Bunken Fukyu-kai, 1978.

[2] I. Goldhirsch. Rapid granular flow. Annual Review of Fluid Mechanics, 35:267, 2003.

[3] I. Goldhirsch. Reaction of the absorber as the mechanism of radiative damping. Powder Technology, 182:130, 2008.

[4] A. Mehta and S.F. Edwards. Satistical machanics of powder mixtures. Physica A, 157:1091-1097, 1989.

[5] S.F. Edwards. Disorder in Condensed Matter Physics. Eds. J. Blackman and J. Taguena. Oxford Univ. Press, Oxford, 1991.

[6] S.F. Edwards. Granular Matter: An Interdisciplinary Approach. Ed. A. Mehta. Springer, NewYork, 1994.

[7] T. Pöschel. Granular gas dynamics. Phys. Rev. Lett., 85:3676-3679, Oct 2000.

[8] Rouyer Florence and Menon Narayanan. Velocity fluctuations in a homogeneous 2d granular gas in steady state. Phys. Rev. Lett., 85:3676-3679, Oct 2000.

[9] Wildman R. D., Huntley J. M., and D. J. Parker. Convection in highly fluidized three-dimensional granular beds. Phys. Rev. Lett., 86:3304-3307, Apr 2001.

[10] Holland, DJ, Müller, CR, Dennis JS, Gladden LF, and Sederman AJ. Spatially resolved measurement of anisotropic granular temperature in gas-fluidized beds. Powder Technology, 182(2):171-181, 2008.

[11] G D'anna, Mayor P, A Barrat, Loreto V, and Franco Nori. Observing brownian motion in vibration-fluidized granular matter. Nature, 424(6951):909-912, 2003.

[12] M. Toda, R. Kubo, and N. Hashitsume. Statistical Physics II: Nonequilibrium Statistical Mechanics vol II. Berlin: Springer, 1985.

[13] Denis J. Evans, E. G. D. Cohen, and G. P. Morriss. Probability of second law violations in shearing steady states. Phys. Rev. Lett., 71:2401-2404, Oct 1993.

[14] G. Gallavotti and E. G. D. Cohen. Dynamical ensembles in nonequilibrium statistical mechanics. Phys. Rev. Lett., 74:2694-2697, Apr 1995.

[15] A. Naert. Experimental study of work exchange with a granular gas: The viewpoint of the fluctuation theorem. EPL (Europhysics Letters), 97(2):20010, 2012. 
Two methods to measure granular gas temperature.

[16] J.-Y. Chastaing, J.-C. Géminard, and A. Naert. Experimental study of energy exchanges between two coupled granular gases. Phys. Rev. E, 94:062110, Dec 2016.

[17] Aumaître, S., Fauve, S., McNamara, S., and Poggi, P. Power injected in dissipative systems and the fluctuation theorem. Eur. Phys. J. B, 19(3):449-460, 2001.

[18] Malte Schmick, Qi Liu, Qi Ouyang, and Mario Markus. Fluctuation theorem for a single particle in a moving billiard: Experiments and simulations. Phys. Rev. E, 76:021115, Aug 2007.

[19] Jean-Yonnel Chastaing, Jean-Christophe Géminard, and Éric Bertin. A statistical physics viewpoint on the dynamics of the bouncing ball. Journal of Statistical Mechanics: Theory and Experiment, 2016(6):063202, 2016.

[20] LF Cugliandolo, J Kurchan, and L Peliti. Energy flow, partial equilibration, and effective temperatures in systems with slow dynamics. PHYSICAL REVIEW E, 55(4):3898-3914, APR 1997.

[21] L Bellon and S Ciliberto. Experimental study of the fluctuation dissipation relation during an aging process. Physica D: Nonlinear Phenomena, 168-169:325-335, 2002. \{VII\} Latin American Workshop on Nonlinear Phenomena.

[22] Bérengère Abou, François Gallet, Pascal Monceau, and Noelle Pottier. Generalized Einstein relation in an aging colloidal glass. Physica A: Statistical Mechanics and its Applications, 387(14):3410 - 3422, 2008.

[23] Chaoming Song, Ping Wang, and Hernán A. Makse. Experimental measurement of an effective temperature for jammed granular materials. Proceedings of the National Academy of Sciences of the United States of America, 102(7):2299-2304, 22005.

[24] S. Joubaud, B. Percier, A. Petrosyan, and S. Ciliberto. Aging and effective temperatures near a critical point. Phys. Rev. Lett., 102:130601, Mar 2009.

[25] Pierre Jop, Juan Ruben Gomez-Solano, Artyom Petrosyan, and Sergio Ciliberto. Experimental study of out-of-equilibrium fluctuations in a colloidal suspension of laponite using optical traps. Journal of Statistical Mechanics: Theory and Experiment, 2009(04):P04012, 2009.

[26] Romain Monchaux, Pierre-Philippe Cortet, Pierre-Henri Chavanis, Arnaud Chiffaudel, François Daviaud, Pantxo Diribarne, and Bérengère Dubrulle. Fluctuation-dissipation relations and statistical temperatures in a turbulent von kármán flow. Phys. Rev. Lett., 101:174502, Oct 2008.

[27] V Grenard, N B Garnier, and A Naert. The fluctuation-dissipation relation on a melde string in a turbulent flow; considerations on a 'dynamical temperature'. Journal of Statistical Mechanics: Theory and Experiment, 2008(09):L09003, 2008.

[28] Jérémie Palacci, Cécile Cottin-Bizonne, Christophe Ybert, and Lydéric Bocquet. Sedimentation and effective temperature of active colloidal suspensions. Phys. Rev. Lett., 105:088304, Aug 2010 . 